\title{
Crude reality versus data manipulation: is the glass always crystal clear?
}

\author{
Heleen M. Oudemans-van Straaten ${ }^{1 *}$ and Jean-Jacques Parienti ${ }^{2,3}$ \\ ๑ 2016 Springer-Verlag Berlin Heidelberg and ESICM
}

A patient with chronic dialysis was admitted to the ICU for septic shock and died. What would have been the outcome if this patient had not been exposed to chronic dialysis prior to septic shock? In the real world, the identical situation never occurs twice at the same time, which creates a $50 \%$ of missing data compared to the counterfactual world, because removing the exposure to see what happens is impossible. The variability of factors associated with exposure and outcome has the potential to confound the variability in the outcome associated with the variability of exposure.

The group of Manish Sood [1] recently reported an important study evaluating the clinical characteristics and in-hospital mortality of patients experiencing septic shock with and without chronic dialysis. The study showed that chronic dialysis patients had a higher mortality than non-dialysis patients. Dialysis patients had higher Acute Physiology and Health Evaluation (APACHE) II scores, more nosocomial infections, dialysis-related infection (central venous catheter-related and peritonitis) and carried different types of pathogens. Furthermore, septic shock in dialysis patients was more often caused by highly resistant organisms. Consequently, both antibiotic inappropriateness and the delay of antimicrobial administration were greater in the dialysis population. After propensity score matching (PSM) [2], mortality was similar between the dialysis and nondialysis group. Which lessons do we learn from the propensity score matching in this study and what does this study teach us about clinical practice?

\section{Propensity score matching and its consequences}

In the Manish Sood study, PSM was done to compensate for several baseline differences in order to explain

\footnotetext{
*Correspondence: hmoudemans@gmail.com

1 Department of Adult Intensive Care, VU University Medical Centre, De Boelelaan 1118, 1081 HZ Amsterdam, The Netherlands

Full list of author information is available at the end of the article
}

whether these differences contributed to the observed higher mortality of dialysis patients. However, PSM does not compensate for the fact that chronic dialysis patients cannot recover a normal kidney function. They also differed from the non-dialysis patients with sepsis regarding acute physiology, the need for invasive dialysis procedures involving disruption of skin and mucosa barriers, type and site of sepsis, causative microorganisms and immunological defence, all contributing to increased mortality. This is clinical reality.

Nevertheless, the difference in mortality disappeared after PSM. This result suggests that chronic dialysis is not an independent risk factor for sepsis death per se, but that the higher APACHE II score and inappropriate antimicrobials were the major contributors to the higher mortality in the dialysis patients (Fig. 1).

\section{APACHE score}

The components of the APACHE II score are acute physiology score, age, chronic health points and (non)elective admission [3]. As a result of inclusion, all admissions were nonelective. Despite the fact that dialysis patients were younger, the combination of acute physiology score and more chronic health points in dialysis patients yielded higher APACHE II scores. Chronic dialysis status partially explained the APACHE II difference, both due to a higher serum creatinine and to chronic dialysis counting as comorbidity. After PSM, APACHE II scores of the non-dialysis group increased and PSM led to the inclusion of sicker non-dialysis patients, either due to a high acute physiology score or to other comorbidities [4].

\section{Inappropriateness of antimicrobial treatment}

Dialysis patients were more likely to receive inappropriate antimicrobial therapy. Inappropriateness seemed to be associated with Candida or fungal infections, uncovered Gram-negatives (Pseudomonas), narrow spectrum of empirical therapy and multiresistant strains. After

\section{照 Springer}




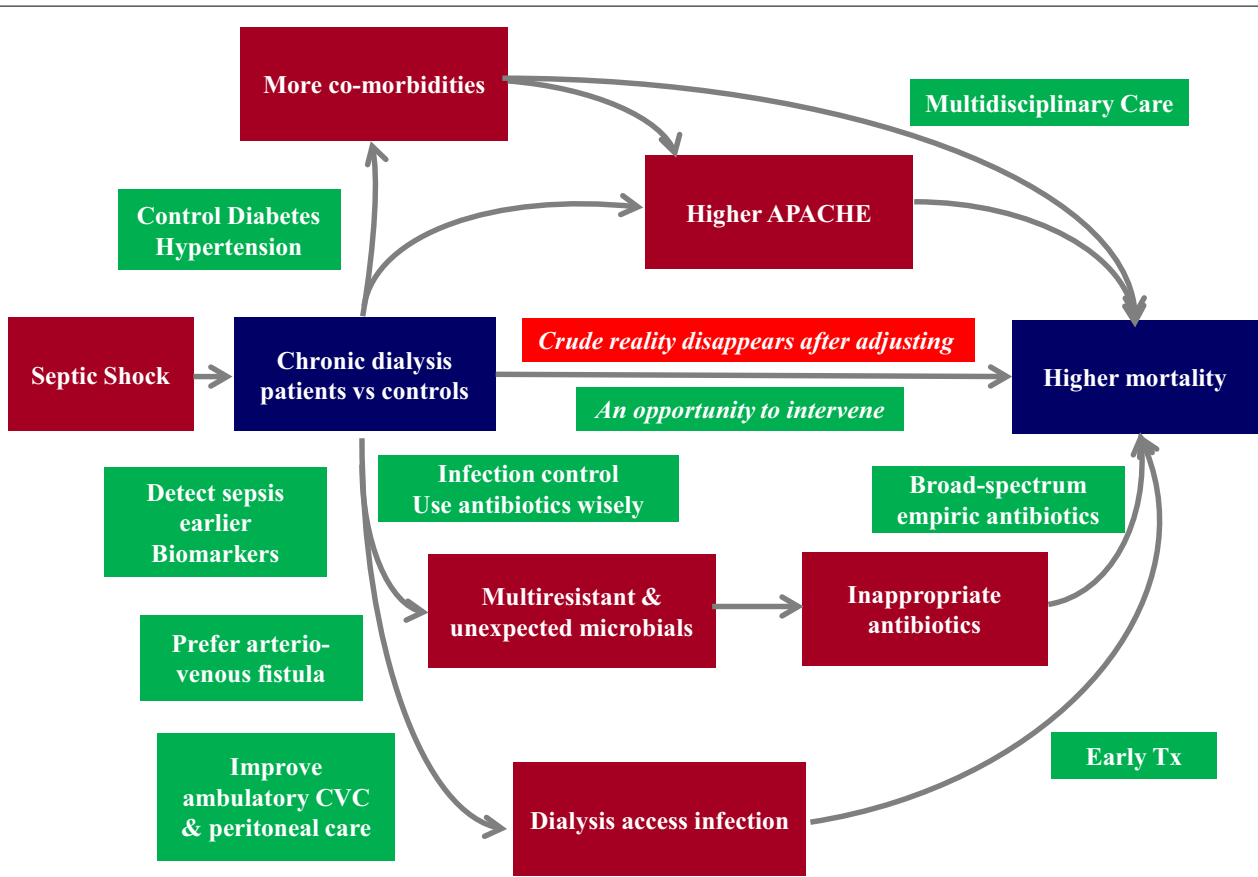

Fig. 1 Relationship between dialysis patients and mortality from septic shock: an opportunity for interventions. APACHE Acute Physiology and Health Evaluation score, Tx treatment

PSM, inappropriateness diminished. Thus, the present results suggest that inappropriateness of antimicrobial treatment partially explains the higher mortality of the dialysis patients. It may also indicate that patients with higher APACHE scores are more likely to have septic shock due to unexpected pathogens not covered by standard empirical protocols, or as the authors mention, that illness severity, age and comorbidity may mask information regarding the presence, site and microbials involved in septic shock.

\section{Time of dying}

More chronic dialysis patients died later, after 1 month, while death in the first week of ICU admission was less in the chronic dialysis population. The difference remained after PSM. Multiple factors could account for this variation. Early dying may be less, as the authors suggest, because a lower initial proinflammatory response could be advantageous causing less intractable shock, but immune suppression hampers microbial killing and increases susceptibility to secondary infections [5]. An alternative hypothesis could be that initial support was more intense in the younger dialysis population than in the older non-dialysis patients with grim comorbidity. Furthermore, chronic dialysis may cause more drug toxicity and difficulties in managing the disturbed fluid and electrolyte homeostasis. Also, their frailty may contribute to weaning failure. Finally, several "time-varying" confounding factors until death were not controlled for here. Nevertheless, the timing of death may have a lower societal value in terms of quality of life, when survival is prolonged within the hospital.

\section{Lessons for daily practice}

The lesson from this work is that chronic dialysis patients have a higher mortality from septic shock than non-dialysis patients. Therefore, more efforts should be done to prevent [6] and detect [7] infection in chronic dialysis patients (Fig. 1). Although an increasing antibiotic resistance cascade is of concern [8], the clinical threshold to initiate early broad-spectrum antimicrobial including fungus infection coverage should be lowered [9].

An aspect not mentioned by this study is the higher risk of inappropriate dosing of antimicrobial treatment causing too low or toxic drug concentrations. During septic shock, the volume of distribution is generally increased, necessitating initial higher dosing, also for chronic dialysis patients, and especially when severity of disease is high [10]. A high loading dose is often not part of standard antibiotic protocols. Too low initial dosing delays appropriate antimicrobial coverage.

In contrast with Sood et al's conclusion in their abstract, we do think that mortality following septic shock is higher in dialysis than in non-dialysis patients, i.e. different. However, PSM elucidated that chronic dialysis is not a risk factor for death per se. While crude 
reality identifies a problem, high-quality large databases combined with in-depth statistical analyses, such as the work performed by the Cooperative Antimicrobial Therapy of Septic Shock Database Research Group discussed here, are indispensable to untangle the pathway of multicausality and ultimately delineate a research agenda for interventions able to improve outcome.

\section{Author details}

${ }^{1}$ Department of Adult Intensive Care, VU University Medical Centre, De Boelelaan 1118, $1081 \mathrm{HZ}$ Amsterdam, The Netherlands. ${ }^{2}$ Unité de Biostatistique et de Recherche Clinique. Pôle de Recherche et d'Epidémiologie Clinique, Centre Hospitalier Universitaire, Caen, France. ${ }^{3}$ Equipe d'Accueil (EA) Groupe de Recherche sur l'Adaptation Microbienne (GRAM) 2.0, Université Caen Normandie, Caen, France.

\section{Compliance with ethical standards}

\section{Conflicts of interest}

The authors declare that they have no conflict of interest.

Received: 4 December 2015 Accepted: 9 December 2015

Published online: 20 January 2016

\section{References}

1. Clark E, Kumar A, Langote A, Lapinsky S, Dodek P, Kramer A et al (2015) Septic shock in chronic dialysis patients: clinical characteristics, antimicrobial therapy and mortality. Intensive Care Med. doi:10.1007/ s00134-015-4147-9
2. Gayat E, Pirracchio R, Resche-Rigon M, Mebazaa A, Mary J-Y, Porcher R (2010) Propensity scores in intensive care and anaesthesiology literature: a systematic review. Intensive Care Med 36:1993-2003

3. Knaus WA, Draper EA, Wagner DP, Zimmerman JE (1985) APACHE II: a severity of disease classification system. Crit Care Med 13:818-829

4. Pittet D, Thiévent B, Wenzel RP, Li N, Gurman G, Suter PM (1993) Importance of pre-existing co-morbidities for prognosis of septicemia in critically ill patients. Intensive Care Med 19:265-272

5. Dalrymple LS, MuY, Nguyen DV, Romano PS, Chertow GM, Grimes B et al (2015) Risk factors for infection-related hospitalization in in-center hemodialysis. Clin J Am Soc Nephrol. doi:10.2215/CJN.03050315

6. Laurin L-P, Harrak H, Elftouh N, Ouimet D, Vallée M, Lafrance J-P (2015) Outcomes of infection-related hospitalization according to dialysis modality. Clin J Am Soc Nephrol 10:817-824

7. Dahaba AA, Rehak PH, List WF (2003) Procalcitonin and C-reactive protein plasma concentrations in nonseptic uremic patients undergoing hemodialysis. Intensive Care Med 29:579-583

8. Bassetti M, De Waele JJ, Eggimann P, Garnacho-Montero J, Kahlmeter G, Menichetti F et al (2015) Preventive and therapeutic strategies in critically ill patients with highly resistant bacteria. Intensive Care Med 41:776-795

9. Parienti JJ, Lucet JC, Lefort A, Armand-Lefèvre L, Wolff M, Caron F et al (2015) Empirical therapies among adults hospitalized for communityacquired upper urinary tract infections: a decision-tree analysis of mortality, costs, and resistance. Am J Infect Control. doi:10.1016/j. ajic.2015.05.033

10. Tsai D, Lipman J, Roberts JA (2015) Pharmacokinetic/pharmacodynamic considerations for the optimization of antimicrobial delivery in the critically ill. Curr Opin Crit Care 21:412-420 